\title{
The proportion of tumour cells is an independent predictor for survival in colorectal cancer patients
}

\author{
NP West', M Dattani', P McShane ${ }^{2}$, G Hutchins', J Grabsch', W Mueller ${ }^{3}$, D Treanor', P Quirke' and \\ H Grabsch ${ }^{*, 1}$
}

'Pathology and Tumour Biology, Leeds Institute of Molecular Medicine, University of Leeds, St James's University Hospital, Leeds LS9 7TF, UK; ${ }^{2}$ Centre for Epidemiology and Biostatistics, Institute of Genetics, Health and Therapeutics, University of Leeds, Leeds LS2 9JT, UK; ${ }^{3}$ Gemeinschaftspraxis Pathologie, Stamberg, Germany

\begin{abstract}
BACKGROUND: The proportion of epithelial and stromal cells in tumours is thought to have an important role in the progression of epithelial malignancy. We aimed to determine whether the relative proportion of tumour (PoT) was related to survival in colorectal cancer.

METHODS: The POT at the luminal surface was measured by point counting using virtual tissue sections in a series of I 45 colorectal cancer cases. The relationship of PoT to clinicopathological parameters including cancer-specific survival was analysed. Modified receiver operating characteristic curves were used to determine the optimum cut off points to dichotomise the data for survival analyses.

RESULTS: Tumours with POT-low ( $\leqslant 47 \%$ ) were associated with significantly lower cancer-specific survival when compared to PoT-high (hazard ratio $(H R)=2.087,95 \% \mathrm{Cl}=1.088-4.003, P=0.024)$. On sub-analysis, the prognostic effect remained significant in colonic tumours $(H R=2.474,95 \% \mathrm{Cl}=1.132-5.408, P=0.019)$ and tumour, node, metastasis stage $\|||$ disease $(H R=3.480,95 \%$ $\mathrm{Cl}=0.325-9.136, P=0.007)$. Multivariate Cox regression analysis demonstrated that PoT was an independent prognostic marker when adjusted for age, $T$ stage, $N$ stage and extramural vascular invasion $(P=0.017)$.

CONCLUSION: This study suggests that a low proportion of tumour cells in colorectal cancer is related to poor cancer-specific survival. A relatively quick, inexpensive and well-established method such as point counting on diagnostic tissue sections could be used to identify a subset of patients who may benefit from adjuvant therapy.

British Journal of Cancer (2010) I 02, I5I9- 1523. doi:I0.1038/sj.bjc.6605674 www.bjcancer.com

Published online 20 April 2010

(c) 2010 Cancer Research UK
\end{abstract}

Keywords: colorectal cancer; proportion of tumour; point counting; virtual slides

Colorectal cancer (CRC) is a common disease and despite significant advances in its treatment, the 5-year overall survival remains around 50\% (Cancer Research UK, 2009). Recent figures show that CRC is now the second commonest cause of cancer related mortality in the United Kingdom with over 16000 deaths occurring annually (Cancer Research UK, 2009). Staging systems such as Dukes (Gabriel et al, 1935) and tumour, node, metastasis (TNM) (Sobin and Wittekind, 1997, pp 66-69) are routinely used to predict prognosis following surgery, however, patients diagnosed at the same stage of disease often have markedly different outcomes (Morris et al, 2007). Current research aims to identify additional prognostic markers that can be used to stratify CRC patients and identify those which may benefit from adjuvant therapy.

Similar to all other malignant epithelial tumours, CRC is composed of carcinoma cells admixed with stromal fibroblasts, lymphatic and vascular channels, and inflammatory cells, often referred to as the tumour microenvironment. This microenviron-

*Correspondence: Dr H Grabsch; E-mail: h.i.grabsch@leeds.ac.uk Received 22 December 2009; revised 25 March 2010; accepted 29 March 2010; published online 20 April 2010 ment is becoming increasingly recognised as having an important role in tumour cell invasion and the ability to metastasise (De Wever and Mareel, 2003).

Very few studies in selected cancer subtypes, such as breast (Baak et al, 1985), lung (Nakajima, 1991; Maeshima et al, 2002; Das Neves Pereira et al, 2004), skin (Breuninger et al, 1997) and prostate cancer (Yanagisawa et al, 2007) have quantified the cellular components of primary tumours and demonstrated that the tumour composition is associated with patient survival. In CRC, the number of stromal myofibroblasts (Tsujino et al, 2007), vimentin expression (Ngan et al, 2007) and degree of stromal desmoplasia (Halvorsen and Seim, 1989; Shepherd et al, 1997; Sis et al, 2005) have been associated with patient prognosis in the past. So far, only one study has suggested that the proportion of tumour cells in CRC may be important (Mesker et al, 2007). However, this result was based upon a relatively small number of cases using qualitative visual estimation of the epithelial component rather than measuring the components objectively by a well-established morphometric method, such as point counting that was first described in the 1940s (Chalkley, 1943) and developed further by Weibel in the 1960s and 70s (Weibel, 1969). 
We hypothesised that the relative proportion of tumour (PoT) determined by objective point counting on virtual (scanned) haematoxylin and eosin-stained slides is related to cancer-specific survival in CRC patients.

\section{MATERIALS AND METHODS}

\section{Patients}

A total of 145 patients who had potentially curative resections for colorectal adenocarcinoma at the Marienhospital, Düsseldorf, Germany between January 1990 and December 1995 were selected for this study. None of the patients had received pre-operative chemotherapy or radiotherapy. The median follow-up time was 4.3 years (interquartile range $=2.2-6.2$ years) and 107 patients $(73.8 \%)$ were alive at the end of the study period.

\section{Clinicopathological data}

Histopathological staging data was obtained from the pathology reports or from slide review by one of the pathologists (WM) and included the site of the tumour, extramural vascular invasion status, maximum depth of invasion ( $\mathrm{pT}$ ), lymph node involvement $(\mathrm{pN})$ and distant metastasis $(\mathrm{pM})$ according to TNM classification, 5 th edition (Sobin and Wittekind, 1997, pp 66-69). In addition, we had access to data regarding patient age at diagnosis and whether or not adjuvant therapy was given.

\section{Measurement of the relative PoT}

Four $\mu \mathrm{m}$ thick haematoxylin and eosin-stained tissue sections prepared according to standard protocols from one tumour block representing the deepest tumour infiltration into the wall and the largest tumour volume were scanned at $\times 40$ magnification with an automated scanning system (Aperio XT, Aperio Technologies, Vista, CA, USA). Using a digital slide viewer (ImageScope v8.0, Aperio Technologies), slides were inspected after scanning and a number of preliminary analyses were carried out on a small number of cases to establish the optimal frequency of points and optimal size of the area required to accurately assess the PoT (data not shown). An area of $9 \mathrm{~mm}^{2}$ was selected from the luminal surface of each case in which the tumour cell density seemed to be greatest after visual inspection. A grid with a systematic random sample of 300 points was then superimposed on the selected area using newly developed virtual graticule software (RandomSpot, University of Leeds, Leeds, UK) to count the number of times the point fell over each of the categories. Large areas of necrosis and mucus at the surface were avoided when selecting the area. The number of necessary measurement points established in our preliminary work was consistent with previous descriptions of the number of points needed to accurately assess PoT (Baak et al, 1991, pp 189-209). The following categories were used in the scoring system: tumour (the point falls onto a viable cancer cell), stroma, tumour lumen, necrosis, vessel, inflammation and noninformative (unclassifiable). One of the authors (MD) was trained by an experienced pathologist (HG) in recognising the different categories and subsequently navigated through each point and categorised the material underneath the point while blinded to the histopathological and survival data (Figure 1). To assess interobserver variation, a random sample of 40 cases were double scored by a second pathologist (GH). As MD and GH agreed in $98.3 \%$ of the counts $(\kappa=0.971)$, double scoring of all counts was felt to be unnecessary. The PoT was expressed as a percentage fraction of all the informative points per case. Each case took approximately $20 \mathrm{~min}$ to score.

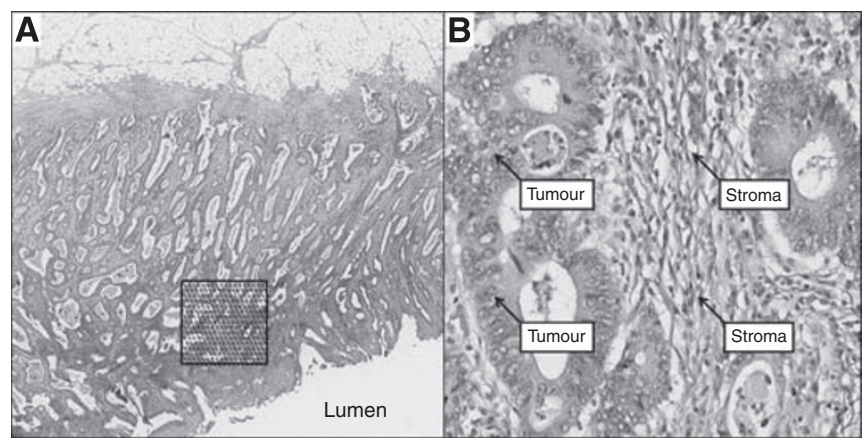

Figure I Morphometric method for establishing the PoT. (A) Selection of a $9 \mathrm{~mm}^{2}$ area at the luminal surface of a haematoxylin and eosin-stained representative section of colorectal cancer. A total of 300 points are randomly inserted into the selected area. (B) Annotation of four individual points comprised of tumour and stroma.

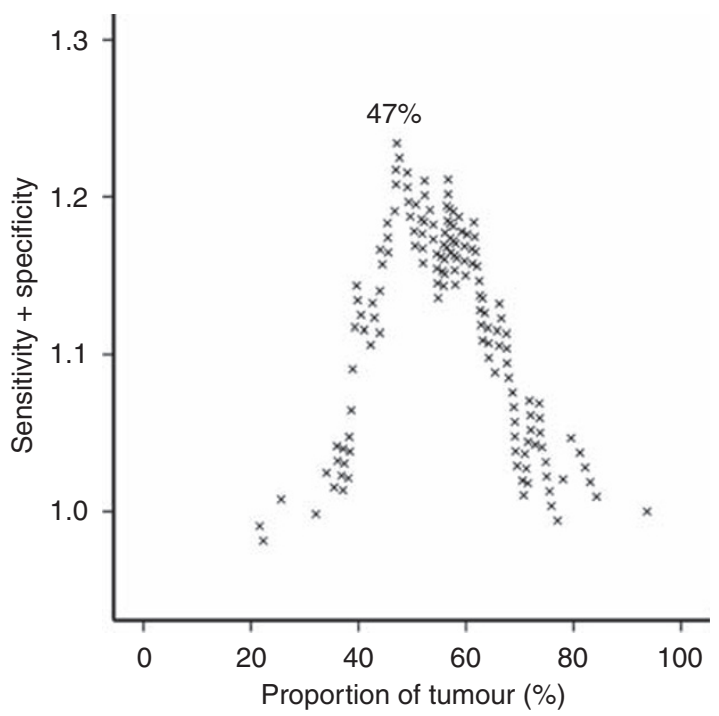

Figure 2 Modified receiver operating characteristic curve used to determine the optimal cut off point for PoT for the subsequent survival analyses. The optimal point is determined from the peak of the curve.

\section{Statistical analyses}

Statistical analyses were performed using the Statistical Package for the Social Sciences (SPSS v15.0, Chicago, IL, USA). Comparisons between PoT and clinicopathological variables were performed using the Mann-Whitney $U$ or Kruskal-Wallis test as appropriate. Correlation analyses were performed using Pearson's correlation coefficients.

The primary endpoint was death attributable to CRC. Cancerspecific survival data were available for all patients. Using a modified receiver operating characteristic curve approach developed by one of the authors (PMcS), the cut off for dichotomisation of PoT with the highest sensitivity and specificity regarding survival prediction was calculated (Figure 2). Using this approach, PoT was classified as either PoT-high ( $>47 \%$ of tumour cells within the tumour) or PoT-low ( $\leqslant 47 \%$ of tumour cells within the tumour).

Patients who died within 30 days after surgery (post-operative mortality) were excluded from the study. Univariate survival analyses were performed using Kaplan-Meier curves (Kaplan and Meier, 1958) and differences between the groups assessed with the 
log-rank test. To assess whether the potential new prognostic marker predicts survival independent from known prognostic markers, such as $\mathrm{pT}, \mathrm{pN}$, extramural blood vessel invasion and age, multivariate survival analyses were performed using the Cox proportional hazards regression model (Cox, 1972). P-values of less than 0.05 were considered to be statistically significant.

\section{Ethical approval}

Ethical approval for the study was granted by the Northern and Yorkshire Research Ethics Committee, Jarrow, UK (unique reference number 08/H0903/62).

\section{RESULTS}

\section{Clinicopathological data}

The median age of patients was 69 years (interquartile range from 61 to 76 years). The remaining clinicopathological data is displayed in Table 1.

Table I Clinicopathological data and its association with the proportion of PoT

\begin{tabular}{|c|c|c|c|c|c|c|c|}
\hline & \multirow{3}{*}{$\begin{array}{c}\text { All cases } \\
n\end{array}$} & \multirow[b]{3}{*}{$\%$} & \multicolumn{4}{|c|}{ Proportion of tumour } & \multirow[b]{3}{*}{$P$-valuc } \\
\hline & & & \multicolumn{2}{|c|}{ High } & \multicolumn{2}{|c|}{ Low } & \\
\hline & & & $n$ & $\%$ & $n$ & $\%$ & \\
\hline \multicolumn{8}{|l|}{ Gender } \\
\hline Male & 58 & 40 & 44 & 40 & 14 & 40 & 1.000 \\
\hline Female & 87 & 60 & 66 & 60 & 21 & 60 & \\
\hline \multicolumn{8}{|c|}{ Tumour location } \\
\hline Colon & 102 & 70 & 81 & 74 & 21 & 60 & 0.125 \\
\hline Rectum & 43 & 30 & 29 & 26 & 14 & 40 & \\
\hline \multicolumn{8}{|c|}{ Adjuvant treatment } \\
\hline Yes & 21 & 15 & 93 & 85 & 31 & 89 & 0.557 \\
\hline No & 124 & 86 & 17 & 15 & 4 & 11 & \\
\hline \multicolumn{8}{|c|}{ Depth of invasion (pT) } \\
\hline PTI & 11 & 8 & 10 & 9 & 1 & 3 & 0.064 \\
\hline PT2 & 27 & 19 & 22 & 20 & 5 & 14 & \\
\hline PT3 & 95 & 66 & 71 & 65 & 24 & 69 & \\
\hline PT4 & 12 & 8 & 7 & 6 & 5 & 14 & \\
\hline \multicolumn{8}{|c|}{ Lymph node status ( $p N$ ) } \\
\hline pNO & 97 & 67 & 75 & 68 & 22 & 63 & 0.650 \\
\hline $\mathrm{pNI}$ & 30 & 21 & 21 & 19 & 9 & 26 & \\
\hline $\mathrm{pN} 2$ & 18 & 12 & 14 & 13 & 4 & 11 & \\
\hline \multicolumn{8}{|c|}{ Distant metastasis (pM) } \\
\hline pMO & 143 & 99 & 109 & 99 & 34 & 97 & 0.391 \\
\hline pMI & 2 & I & । & I & । & 3 & \\
\hline \multicolumn{8}{|l|}{ TNM stage } \\
\hline I & 33 & 23 & 27 & 25 & 6 & 17 & 0.294 \\
\hline$\|$ & 62 & 43 & 47 & 43 & 15 & 43 & \\
\hline$\|$ & 48 & 33 & 35 & 32 & 13 & 37 & \\
\hline IV & 2 & I & । & । & । & 3 & \\
\hline \multicolumn{8}{|c|}{ Extramural vascular invasion } \\
\hline No & 140 & 97 & 106 & 96 & 34 & 97 & 0.826 \\
\hline Yes & 5 & 3 & 4 & 4 & I & 3 & \\
\hline
\end{tabular}

Abbreviations: PoT = proportion of tumour; TNM = tumour, node, metastasis. Staging data was obtained using TNM, 5th edition (Sobin and Wittekind, 1997, pp 66-69). Clinicopathological data and its association with the proportion of tumour (PoT) which has been dichotomised into PoT-high ( $>47 \%$ of tumour cells) and PoT-low ( $\leqslant 47 \%$ of tumour cells).

\section{Relative proportion of tumour (PoT)}

The PoT value followed a normal distribution across the series ranging from 21.6 to $84.3 \%$ (see Figure 3 ). The median PoT value was $57.1 \%$ (interquartile range from 47.4 to $66.4 \%$ ). The relationship between high and low PoT with clinicopathological data is shown in Table 1. There was no significant correlation between PoT and any of the clinicopathological variables, however, a low PoT was weakly related to pT stage rising from $9 \%$ in pT 1 to $42 \%$ in pT4. The frequency of low PoT was higher in cancers of the rectum compared with cancers of the colon, however, this was not statistically significant (33 vs 21\%, $P=0.125$ ). The PoT value was strongly inversely correlated with the proportion of stroma within the tumour $(r=-0.913, P<0.0001)$.

\section{Survival analyses}

The results of the survival analyses for all cases are shown in Table 2. On univariate analysis, PoT-low was associated with poorer cancer-specific survival (hazard ratio $(H R)=2.087,95 \%$ confidence interval $(\mathrm{CI})=1.088-4.003, P=0.024$, Figure 4$)$. This was also significant when follow-up was censored at 3 years $(\mathrm{HR}=2.878,95 \% \mathrm{CI}=1.369-6.049, \quad P=0.003)$ and 5 years $(\mathrm{HR}=2.083, \quad 95 \% \quad \mathrm{CI}=1.066-4.072, \quad P=0.028)$. Multivariate

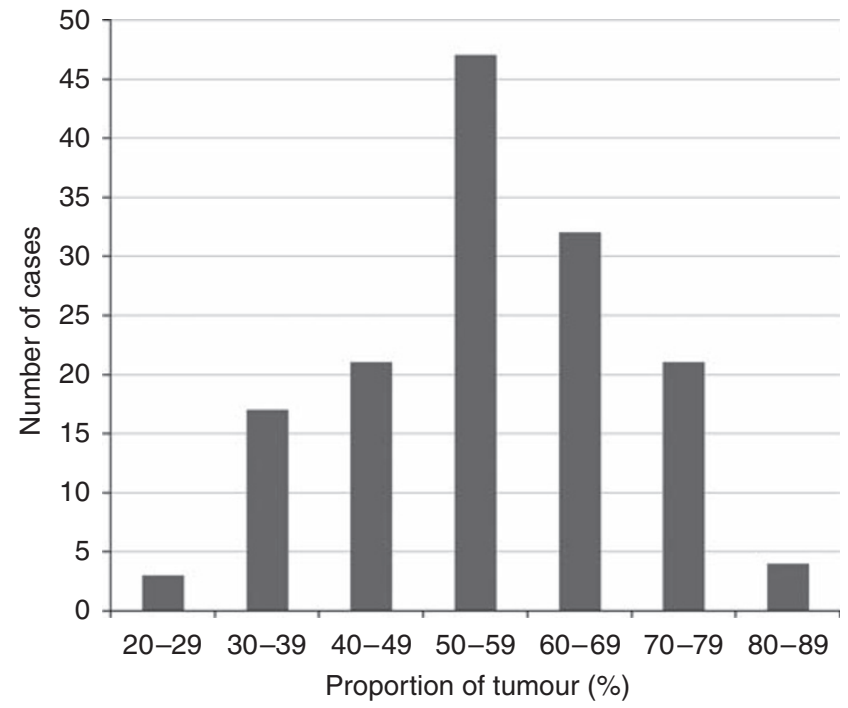

Figure 3 The distribution of the proportion of tumour cells across the colorectal cancer patient population.

Table 2 Univariate survival data obtained with the log-rank test

\begin{tabular}{|c|c|c|c|c|}
\hline & \multicolumn{3}{|c|}{ Univariate data } & \multirow{2}{*}{$\begin{array}{c}\text { Multivariate data } \\
\text { P-value }\end{array}$} \\
\hline & Chi-square & Df & $P$-value & \\
\hline Age (per 10-year increase) & 11.824 & 4 & 0.019 & 0.019 \\
\hline Gender & 1.247 & I & 0.264 & - \\
\hline Tumour location & 0.061 & I & 0.805 & - \\
\hline Adjuvant treatment given & 0.783 & 1 & 0.376 & - \\
\hline Depth of invasion (pT) & 20.974 & 3 & $<0.0001$ & 0.070 \\
\hline Lymph node status ( $\mathrm{pN}$ ) & 6.283 & 2 & 0.043 & 0.011 \\
\hline Distant metastasis (pM) & 0.336 & I & 0.562 & - \\
\hline Extramural vascular invasion & 5.076 & i & 0.024 & 0.016 \\
\hline Proportion of tumour & 5.129 & I & 0.024 & 0.017 \\
\hline
\end{tabular}

Abbreviation: $\mathrm{Df}=$ degrees of freedom. Univariate survival data obtained with the log rank test for the various clinicopathological variables, along with the relevant multivariate $P$-values obtained using a Cox proportional hazards regression analysis. 
analysis confirmed that PoT-low was an independent poor prognostic marker when the model was adjusted for age, $\mathrm{pT}$ stage, pN stage and extramural vascular invasion $(P=0.017)$.

Subgroup analyses by tumour location demonstrated that PoT is a significant prognostic factor in colonic cancers $(\mathrm{HR}=2.474,95 \% \mathrm{CI}=1.132-5.408, P=0.019)$ but not in rectal cancers $(\mathrm{HR}=1.693,95 \% \mathrm{CI}=0.516-5.562, P=0.380)$. This result was confirmed on multivariate analysis for both colonic $(\mathrm{HR}=2.703,95 \% \mathrm{CI}=1.183-6.175, P=0.018)$ and rectal cancers $(\mathrm{HR}=1.610,95 \% \mathrm{CI}=0.471-5.505, P=0.448)$. Table 3 shows the survival analyses according to the TNM stage of disease. The prognostic effect of PoT remained in TNM stage III disease on both univariate $(\mathrm{HR}=3.480,95 \% \mathrm{CI}=1.325-9.136, P=0.007)$ and multivariate analysis $\quad(\mathrm{HR}=3.121, \quad 95 \% \quad \mathrm{CI}=1.091-8.929$, $P=0.034)$.

\section{DISCUSSION}

Malignant tumours such as CRC are supported by a rich network of stroma that undergoes varying degrees of modification after epithelial cell invasion including desmoplasia (Hewitt et al, 1993), angiogenesis and inflammatory cell infiltration (De Wever and Mareel, 2003). The composition of tumours varies dramatically among patients and has previously been linked to differential survival in the breast (Baak et al, 1985), lung (Nakajima, 1991; Maeshima et al, 2002; das Neves Pereira et al, 2004), skin

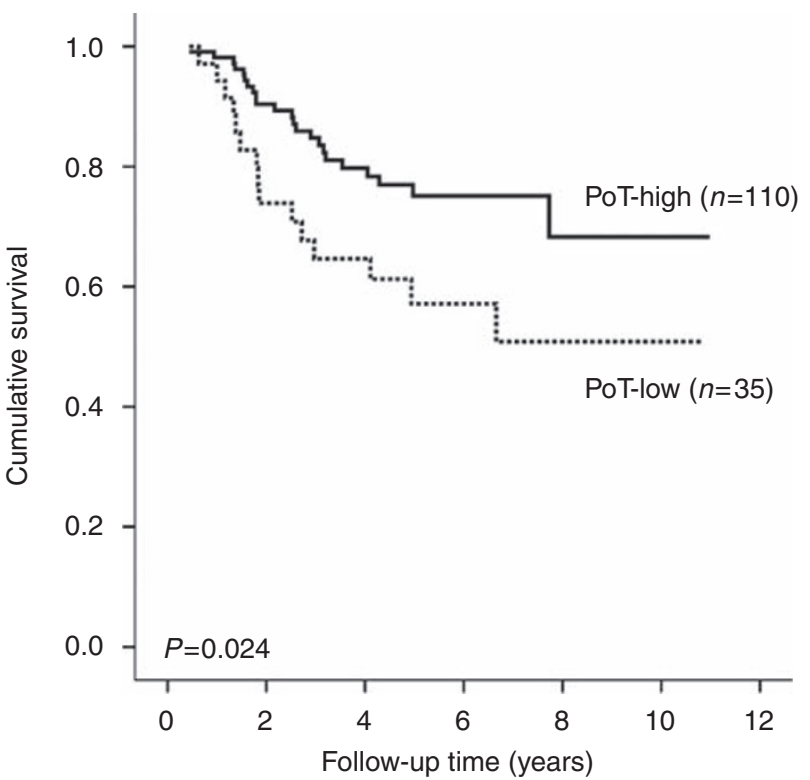

Figure 4 Kaplan-Meier survival curve after stratification by PoT. Hazard ratio for PoT-low $=2.087$ ( $95 \%$ confidence interval $=1.088-4.003$ ).
(Breuninger et al, 1997), prostate cancer (Yanagisawa et al, 2007) and CRC (Halvorsen and Seim, 1989; Shepherd et al, 1997; Sis et al, 2005; Ngan et al, 2007; Tsujino et al, 2007). Most of these studies demonstrate that a greater proportion of stroma or an exaggerated desmoplastic response is associated with poorer patient outcomes (Jass, 1986; Roncucci et al, 1996). Studies in human colon cancer cell lines have shown that organ-specific fibroblasts can directly influence the ability of the tumour cells to invade through the production of factors such as collagenases (Fabra et al, 1992). Molecular studies have shown that specific chromosomal aberrations are related to the proportion of tumour in CRC and therefore may affect tumour-stroma interactions (Fijneman et al, 2007). Quantitative methods to evaluate desmoplasia including computer-assisted image analysis have already been shown to improve reproducibility of the assessment and have confirmed a relationship to survival (Sis et al, 2005; Tsujino et al, 2007).

Not surprisingly, our study demonstrated that the proportion of viable tumour cells within a tumour is strongly correlated to the proportion of stroma. Using quantitative point counting on virtual tissue sections, we have shown that a low proportion of malignant epithelial cells within a given CRC is independently associated with worse cancer-specific survival. The prognostic effect of PoT seemed to be more significant in colonic cancers and TNM stage III disease than rectal cancers and TNM stages I and II, although due to the small numbers involved in these subgroup analyses the results must be viewed with caution and need to be confirmed in a larger series of cases.

There are several theories as to why a low PoT (and therefore high proportion of stroma) within a tumour may infer a poor prognosis. First, it has been hypothesised that tumours with a greater proportion of reactive stroma are able to produce more growth factors thus increasing the overall tumour burden (De Wever and Mareel, 2003). Second, it has been suggested that the relative amount of desmoplastic fibrosis may have a role in reducing the accessibility of tumours to the immune response (Kouniavsky et al, 2002) by encapsulating the malignant cells and preventing their destruction (Liotta et al, 1983). However, one must consider that PoT may reflect the stage of disease, and our study suggests a weak correlation between PoT-low and pT stage, which is in concordance with one previous study in which low PoT was seen in $8 \%$ of stage I patients and in up to $69 \%$ of stage III patients (Mesker et al, 2007).

Morphometrical analysis enables accurate quantification of various tissue components when compared with qualitative systems. The traditional method of using optical graticules on conventional glass slides has limited flexibility. Using software that can insert any number of sampling points within a systematic grid onto a virtual slide (Treanor et al, 2008), scores can be entered and saved as an electronic file for further analysis. Although we have used primary resection material in this study, analysis of stromal grade has been shown to be prognostic on biopsy material from the prostate (Yanagisawa et al, 2007) and our technique could potentially be used on pre-operative biopsies of CRC.

Table 3 Univariate survival data obtained with the log rank test according to TNM stage for PoT-high vs PoT-low

\begin{tabular}{|c|c|c|c|c|c|c|}
\hline & \multirow[b]{2}{*}{$n(\%)$} & \multirow[b]{2}{*}{ Median PoT (IQR) } & \multicolumn{3}{|c|}{ Univariate data } & \multirow{2}{*}{$\begin{array}{c}\text { Multivariate data } \\
\text { P-value }\end{array}$} \\
\hline & & & Chi-square & Df & $P$-value & \\
\hline TNM stage I & $33(23)$ & $57(5 \mid-64)$ & 0.116 & 1 & 0.734 & 0.554 \\
\hline TNM stage ॥ & $62(43)$ & $58(50-68)$ & 1.013 & I & 0.314 & 0.230 \\
\hline TNM stage III & $48(33)$ & $55(45-68)$ & 7.240 & i & 0.007 & 0.034 \\
\hline
\end{tabular}

Abbreviations: $\mathrm{Df}=$ degree of freedom; $\mathrm{IQR}=$ interquartile range; $\mathrm{PoT}=$ proportion of tumour; $\mathrm{TNM}=$ tumour, node, metastasis classification. Also shown are the multivariate $P$-values obtained using a Cox proportional hazards regression analysis (adjusted for age and extramural vascular invasion). TNM stage IV is not included as only two cases fell into this category. 
We and others have observed significant heterogeneity in the PoT within individual tumours (Mesker et al, 2007). In this study, we selected an area at the luminal surface to allow future investigation to determine whether the results may be extrapolated to diagnostic biopsy material. It is possible that different results may be acquired if the measurements were performed in different areas of the tumour, for example, the centre or the advancing edge. In breast cancer, some studies have shown a better prognosis with higher PoT at the tumour periphery (Baak et al, 1985), whereas others have suggested an inverse relationship when assessing the whole tumour (Tanaka et al, 2004).

Much of the recent research into optimising CRC patient management has focussed on identifying (i) prognostic markers that allow us to determine which patients may benefit from adjuvant therapy and (ii) predictive markers which predict the response of individual patients to specific therapeutic regimens. The identification of patients with a poor prognosis based on an estimate of PoT obtained through simple, relatively inexpensive morphometrical measurements could easily be transferred into routine diagnostic practice according to our own experience. On the basis of the results of our present study, we hypothesise that patients with PoT-low may be more likely to respond to agents directed at inhibiting the crosstalk between stromal and epithelial cells, an area that clearly warrants further investigations.

\section{ACKNOWLEDGEMENTS}

NPW and PQ are funded by Yorkshire Cancer Research.

\section{REFERENCES}

Baak JPA, Van Dop H, Kurver PHJ, Hermans J (1985) The value of morphometry to classic prognosticators in breast cancer. Cancer 56: $374-382$

Baak JPA, Langley FA, Hermans J (1991) Classification and prognosis for new cases: some aspects of univariate and multivariate analysis. In Manual of Quantitative Pathology in Cancer Diagnosis and Prognosis Baak JPA (ed), pp 189-209. Springer-Verlag: Berlin

Breuninger H, Schaumburg-Lever G, Holzschuh J, Horny HP (1997) Desmoplastic squamous cell carcinoma of skin and vermilion surface. A highly malignant subtype of skin cancer. Cancer 79: 915-919

Cancer Research UK (2009) (Accessed March 2009) Cancer Stats. http:// info.cancerresearchuk.org/cancerstats/types/ bowel/

Chalkley HW (1943) Methods for quantitative morphological analysis of tissue. J Natl Cancer Inst 4: 47-53

Cox DR (1972) Regression models and life tables. J Royal Stat Soc Series B 34: $187-220$

Das Neves Pereira JC, da Silva AG, Soares F, Ab'Saber AM, Schmidt A, Rodrigues OR, Garippo A, Capelozzi M, de Campos JR, Takagaki T, Jatene FB, Martins S, Capelozzi VL (2004) Nuclear and environment morphometric profile in tumor size and nodal metastasis of resected typical pulmonary carcinoid. Pathol Res Pract 200: 459-467

De Wever O, Mareel M (2003) Role of tissue stroma in cancer cell invasion. J Pathol 200: 429-447

Fabra A, Nakajima M, Bucana CD, Fidler IJ (1992) Modulation of the invasive phenotype of human colon carcinoma cells by organ-specific fibroblasts of nude mice. Differentiation 52: 101-110

Fijneman RJ, Carvalho B, Postma C, Mongera S, van Hinsbergh VW, Meijer GA (2007) Loss of 1p36, gain of 8q24, and loss of 9q34 are associated with stroma percentage of colorectal cancer. Cancer Lett 258: 223-229

Gabriel WB, Dukes C, Bussey HJR (1935) Lymphatic spread in cancer of the rectum. Br J Surgery 23: 395-413

Halvorsen TB, Seim E (1989) Association between invasiveness, inflammatory reaction, desmoplasia and survival in colorectal cancer. J Clin Pathol 42: $162-166$

Hewitt RE, Powe DG, Carter I, Turner DR (1993) Desmoplasia and its relevance to colorectal tumour invasion. Int J Cancer 53: 62-69

Jass J (1986) Lymphocytic infiltration and survival in rectal cancer. J Clin Pathol 39: $585-589$

Kaplan EL, Meier P (1958) Nonparametric estimation from incomplete observations. J Am Stat Assoc 53: 457-481

Kouniavsky G, Khaikin M, Zvibel I, Zippel D, Brill S, Halpern Z, Papa M (2002) Stromal extracellular matrix reduces chemotherapy-induced apoptosis in colon cancer cell lines. Clin Exp Metastasis 19: 55-60

Liotta LA, Rao CN, Barsky SH (1983) Tumor invasion and the extracellular matrix. Lab Invest 49: 636-649
Maeshima AM, Niki T, Maeshima A, Yamada T, Kondo H, Matsuno Y (2002) Modified scar grade. A prognostic indicator in small peripheral lung adenocarcinoma. Cancer 95: 2546-2554

Mesker WE, Junggeburt JMC, Szuhai K, de Heer P, Morreau H, Tanke HJ, Tollenaar RAEM (2007) The carcinoma-stromal ratio of colon carcinoma is an independent factor for survival compared to lymph node status and tumour stage. Cell Oncol 29: 387-398

Morris EJ, Maughan NJ, Forman D, Quirke P (2007) Who to treat with adjuvant therapy in Dukes B/stage II colorectal cancer? The need for high quality pathology. Gut 56: $1419-1425$

Nakajima I (1991) Immunohistochemical study of the extracellular matrix in non-small cell lung cancer: relation to lymph node metastasis and prognosis. Hokkaido Igaku Zasshi 66: 356-368

Ngan CY, Yamamoto H, Seshimo I, Tsujino T, Man-i M, Ikeda J-I, Konishi K, Takemasa I, Ikeda M, Sekimoto M, Matsuura N, Monden M (2007) Quantitative evaluation of vimentin expression in tumour stroma of colorectal cancer. Br J Cancer 96: 986-992

Roncucci L, Fante R, Losi L, DiGregorio C, Micheli A, Benatti P, Madenis N, Ganazzi D, Cassinadri MT, Lauriola P, Ponz de Leon M (1996) Survival for colon and rectal cancer in a population based cancer registry. Eur J Cancer 32: 295-302

Shepherd NA, Baxter KJ, Love SB (1997) The prognostic importance of peritoneal involvement in colonic cancer: a prospective evaluation. Gastroenterology 112: 1096 - 1102

Sis B, Sarioglu S, Sokmen S, Sakar M, Kupelioglu A, Fuzun M (2005) Desmoplasia measured by computer assisted image analysis: an independent prognostic marker in colorectal cancer. J Clin Pathol 58: 32-38

Sobin LH, Wittekind Ch (1997) TNM Classification of Malignant Tumours 5th edn, pp 66-69. Wiley-Liss: New York

Tanaka K, Yamamoto D, Yamada M, Okugawa H (2004) Influence of cellularity in human breast cancer. The Breast 13: $334-340$

Treanor D, Dattani M, Quirke P, Grabsch H (2008) Systematic random sampling with virtual slides: a new software tool for tissue research. Abstract J Pathol 216(suppl 1): s43

Tsujino T, Seshimo I, Yamamoto H, Ngan CY, Ezumi K, Takemasa I, Ikeda M, Sekimoto M, Matsuura N, Monden M (2007) Stromal myofibroblasts predict disease recurrence for colorectal cancer. Clin Cancer Res 13: $2082-2090$

Weibel ER (1969) Stereological principles for morphometry in electron microscopic cytology. Int Rev Cytol 26: 235-302

Yanagisawa N, Li R, Rowley D, Liu H, Kadmon D, Miles BJ, Wheeler TM, Ayala GE (2007) Stromogenic prostatic carcinoma pattern (carcinomas with reactive stromal grade 3 ) in needle biopsies predicts biochemical recurrence-free survival in patients after radical prostatectomy. Hum Pathol 38: $1611-1620$ 